\title{
Moderate Pulsed Electric Field Enhances Fermentation Capacity and Induces Stress Responses in Saccharomyces cerevisiae
}

\author{
Nanjiao Ying ${ }^{1,2 *}$, Wenjia Liu ${ }^{1}$, Chengkai Fan ${ }^{2}$, Qiusi Mo ${ }^{1}$, Shanshan $\mathrm{Li}^{1}$ and Lei Zhu ${ }^{1,2}$ \\ ${ }^{1}$ College of Automation, Hangzhou Dianzi University, 310018, China \\ ${ }^{2}$ Institute of Biomedical Engineering, Hangzhou Dianzi University, 310018, China \\ *For correspondence: yingnj@hdu.edu.cn \\ Received 24 August 2020; Accepted 15 September 2020; Published 10 December 2020
}

\begin{abstract}
Saccharomyces cerevisiae has always gained a huge amount of attention due to its extensive and enormous application value. Various methods are used to optimize the fermentation characteristics of S. cerevisiae. In this work, we investigated the fermentation capacity and stress responses on a physiological level of $S$. cerevisiae which affected by moderate pulsed electric field (PEF, 1 to $3 \mathrm{kV} \cdot \mathrm{cm}^{-1}$ ). The fermentation capacity was illustrated by glucose consumption and metabolites yield (ethanol and glycerol). The expressions of ten glycolysis-associated genes were studied to elucidate the fermentative processes. The results showed that fermentation capacity of $S$. cerevisiae was promoted by moderate PEF treatments, with higher glucose consumption and ethanol yield. Yeast cells also gained a faster growth rate under constant PEF stimulation. Analysis of gene expression involved in glycolytic pathway showed that moderate PEF treatments induced a higher glycolytic flux, especially in terms of synthesis of ethanol. It would be a promising technique for PEF to enhance fermentation capacity and growth of $S$. cerevisiae. (C) 2021 Friends Science Publishers
\end{abstract}

Keywords: Saccharomyces cerevisiae; Pulsed Electric Field (PEF); Fermentation; Stress responses; Ethanol yield; Glucose consumption

\section{Introduction}

Saccharomyces cerevisiae is used wildly in fermentation fields, as in wine-making or production of various high additional value biochemicals such as ethanol, glycerol. Therefore, fermentation of $S$. cerevisiae has always received a huge amount of attention to academic and industrial groups. Currently, it is popular to use various technologies to enhance reproduction stability and metabolic activity of fermenting microorganisms, which almost determine production efficiency and quality of fermentation. For example, ultrasonic or magnetic fields can be used to assist the fermenting process by enhancing metabolite yield and growth (Santos et al. 2010; Ojha et al. 2017). Metabolic engineering has been presented as promising solution, it is necessary to ensure growth rate, titer and yield (Semkiv et al. 2017; Lalwani et al. 2018). Various potential approaches were explored to enhance the fermentation performance with considering factors such as usability, stability and environment-friendly.

Pulsed electric field (PEF) has been normally used as a non-thermal sterilization technique using low energy requirements to inactivate microorganisms without destroying quality and flavor of the food and medicine (Zhao et al. 2019). PEF is usually applied for short duration $(\mu \mathrm{s})$ by high intensity electric fields $\left(1\right.$ to $\left.50 \mathrm{kV} \cdot \mathrm{cm}^{-1}\right)$. The mechanism of PEF was commonly known as bacterial inactivation caused by irreversible membrane rupture (Garner 2019). PEF also could modify the functional groups on proteins located on cell membrane directly or indirectly (Hristov et al. 2018). Application values of PEF have received growing attention in recent years, not just limited to food safety. For instance, PEF was found to be a viable elicitor for stimulating secondary metabolite biosynthesis in plant cell cultures (Cai et al. 2011). It was also reported that PEF could enhance activity of enzymes and improve the growth of Lactobacillus acidophilus and Lactobacillus bulgaricus LB-12 (Najim and Aryana 2013; Martens et al. 2020). Proposed that PEF showed a higher efficiency on enhancing the activity of anammox bacteria compared with ultrasound and magnetic field (Yin et al. 2015). The fermentative process of $S$. cerevisiae is also performed by a series of enzymatic catalytic reactions against yeast cells. It is speculated that the PEF might play an active role on fermentation of $S$. cerevisiae under appropriate conditions (Mattar et al. 2015). There are still few studies about direct effects of moderate PEF on physiological behavior of $S$. cerevisiae. The underlying molecular mechanism of PEF on fermentation cells is also poorly reported.

To cite this paper: Ying N, W Liu, C Fan, Q Mo, S Li, L Zhu (2021). Moderate pulsed electric field enhances fermentation capacity and induces stress responses in Saccharomyces cerevisiae. Intl J Agric Biol 25:160-164 
The aim of this study is to investigate the effects of moderate PEF ( 1 to $3 \mathrm{kV} \cdot \mathrm{cm}^{-1}$ ) on the growth and fermentation kinetics to $S$. cerevisiae. We studied the stress responses mechanism by analyzing changes of the expression level of ten genes involved in glycolysis pathway using the reverse transcription-quantitative PCR (RT-qPCR) method. The results revealed that moderate PEF can be applied to promote the fermentation of S. cerevisiae.

\section{Materials and Methods}

\section{Strains, media and inoculum culture}

S. cerevisiae strain RV100, widely used commercial yeast, was purchased from the Angel company (Angel, Yichang, China). The active dry yeast was rehydrated in $5 \%$ glucose solution at $30^{\circ} \mathrm{C}$ for $30 \mathrm{~min}$ and spread on YPD medium plates, then incubated at $30^{\circ} \mathrm{C}$. Single colonies from the agar plate were incubated in $5 \mathrm{~mL}$ YPD broth and culturing at $30^{\circ} \mathrm{C}$ with orbital shaking $(200 \mathrm{rpm})$. The cells were then collected and washed with a phosphate buffered saline (PBS) solution. The initial yeast suspension $\left(\mathrm{OD}_{600}=0.8\right)$ for the fermentation trials was prepared by inoculating cells into synthetic medium ( $8 \%$ glucose, $1 \%$ tryptone, $0.5 \% \mathrm{w} / \mathrm{v}$ yeast extract, $\left.0.1 \%\left(\mathrm{NH}_{4}\right)_{2} \mathrm{SO}_{4}, 0.1 \% \mathrm{MgSO}_{4} \cdot 7 \mathrm{H}_{2} \mathrm{O}, 0.1 \% \mathrm{KH}_{2} \mathrm{PO}_{4}\right)$.

\section{PEF treatment and fermentation}

In this work, a constant treatment protocol was designed to investigate the effects of PEF on $S$. cerevisiae (Fig. 1). The experiments were operated on a cylinder glass bioreactor, which exposed to the PEF treatment chamber. The treatment system contains a circulation pump and temperature controller. The initial yeast suspension (5\% v/v) was inoculated to $300 \mathrm{~mL}$ fermentation media in the bioreactor under PEF processing conditions, while pulse width is $5 \mu \mathrm{s}$, frequency is $10 \mathrm{~Hz}$. The treatment chamber and bioreactor was also disinfected by $75 \%$ alcohol and washed by sterile double distilled water. All experiments were performed in triplicate.

\section{Analysis methods of fermentation kinetics}

Growth dynamic of yeast was monitored by measuring $\mathrm{OD}_{600}$. The dry weight biomass was determined by weighing the cells from suspensions, which washed twice with sterilized water, and then kept at $70^{\circ} \mathrm{C}$ until reaching a constant weight in tubes. Fermentation performance was estimated by measuring the residual glucose, ethanol and glycerol in medium substrate. The concentrations of glucose, ethanol and glycerol were determined by HPLC according to the (Seong et al. 2017). The HPLC column system (Shimadzu, Japan) was set at a flow rate of 0.8 $\mathrm{mL} \cdot \mathrm{min}^{-1}$ with $65^{\circ} \mathrm{C}$ on column.

\section{RNA extraction and cDNA synthesis}

Cells were collected by centrifuged $\left(5000 \mathrm{~g}, 4^{\circ} \mathrm{C}, 5 \mathrm{mins}\right)$ and washed twice with PBS buffer (pH7.2). After grinding with liquid nitrogen, total RNA was extracted from $2 \times 10^{8}$ cells using TransZol Up RNA kit (TransGen). cDNA was synthesized on a Thermal Cycler (Bio-Rad S1000) with PrimeScript $^{\mathrm{TM}}$ RT kit (Takara). All operations were in accordance with the protocol specified by the kit supplier. Qualitative and quantitative analysis of RNA and cDNA were measured by NanoDrop (Thermo Scientific). For each PEF condition, equal RNA amounts were pooled from three independent tests.

\section{Primers and quantitative PCR}

All primers were designed by Primer Premier 5.0 based on the sequences achieved from GenBank (Table 1). Reactions were performed in 96-well plates on a Real-Time QPCR System (Agilent Mx3005P). Baseline and threshold values were automatically determined by the MxPro3005 software. The $2^{-\Delta \Delta C T}$ method was used to calculate the relative expression levels.

\section{Statistical analysis}

Statistical analysis was performed using StatSoft STATISTICA (Statistica, Inc.). Results are reported as mean \pm standard deviation.

\section{Results}

\section{Effects of PEF on fermentation and growth of $S$. cerevisiae}

To investigate the effects of moderate PEF on physiological behavior of $S$. cerevisiae, the constant PEF treatment was applied to initial yeast suspensions. The effects of PEF on the growth dynamics and fermentation kinetics of $S$. cerevisiae were investigated. The selection of PEF parameters was based on extensive preliminary trials for the purpose of avoiding noticeable damage of yeast population caused by PEF. The growth dynamics of $S$. cerevisiae was investigated by examining dry cell biomass and medium absorbance of $\mathrm{OD}_{600}$, which showed in Fig. 2. The weight of dry cell biomass was enhanced $20.6 \%\left(1 \mathrm{kV} \cdot \mathrm{cm}^{-1}\right)$ and $32.4 \%$ (3 $\mathrm{kV} \cdot \mathrm{cm}^{-1}$ ) respectively than those of control samples.

Glucose is the one of the most important nutrient sources for yeast fermentation. The metabolic activity of yeast cells was evaluated by monitoring the uptake of glucose. The residual glucose concentration is presented in Fig. 3a. At the initial stage of fermentation $(0 \sim 3 \mathrm{~h})$, the treated sample $\left(1 \mathrm{kV} \cdot \mathrm{cm}^{-1}\right)$ showed around 1.79 times of glucose consumption than control sample, which it showed around 1.78 times under $3 \mathrm{kV} \cdot \mathrm{cm}^{-1} \mathrm{PEF}$ treatment. It showed that the glucose consumption reached the fastest rate at $9 \mathrm{~h}$ after PEF treatment $\left(1 \mathrm{kV} \cdot \mathrm{cm}^{-1}\right)$, while the control samples reached at $12 \mathrm{~h}$ after treatment. After $12 \mathrm{~h}$, the residual glucose in treated samples were $4.23 \%\left(1 \mathrm{kV} \cdot \mathrm{cm}^{-1}\right)$ and $11.19 \%\left(3 \mathrm{kV} \cdot \mathrm{cm}^{-1}\right)$ lower than those in control samples. During fermentation progress, the residual glucose content was lower in PEF-treated samples than control ones. 
Table 1: Genes and Primers used in the RT-qPCR analyses

\begin{tabular}{|c|c|c|c|}
\hline Genes & ID & Forward and reverse primer & Description \\
\hline \multirow[t]{2}{*}{ ACT1 } & 850504 & 5'-CCA AGA CAC CAA GGT ATC ATG GTC G-3' & actin, structural protein \\
\hline & & 5'-CGG AAG AGT ACA AGG ACA AAA CGG C-3' & \\
\hline \multirow[t]{2}{*}{$G P D 2$} & 854095 & 5'-AAG ATC GGA CTC TGC CGT GTC AAT T-3' & NAD-dependent glycerol 3-phosphate dehydrogenase \\
\hline & & 5'-ACG TGG CCT TGC AAT TGT TTG ACT A-3' & \\
\hline \multirow[t]{2}{*}{$P D C 1$} & 850733 & 5'-AAG GTA TGA GAT GGG CTG GTA ACG C-3' & pyruvate decarboxylase isoenzyme (major one) \\
\hline & & 5'-TGA AGT CAC CGT TAC CCA AGG TGT G-3' & \\
\hline \multirow[t]{2}{*}{ PDC6 } & 852978 & 5'-GCT ACC AGG CGA CTT CAA CTT GT-3' & pyruvate decarboxylase (Minor isoform) \\
\hline & & 5'-TGA GAT ATT GGC GGA CAT TCT GTG A-3' & \\
\hline \multirow[t]{2}{*}{$A L D 4$} & 854556 & 5'-CGT GTT GAA GAC TGC CGA ATC CA-3' & Mitochondrial aldehyde dehydrogenase \\
\hline & & 5'-CGC ACA ACA GAC CTC ACC AGA ATT-3' & \\
\hline \multirow[t]{2}{*}{$A L D 6$} & 856044 & 5'-AAC TTC ACC ACC TTA GAG CCA ATC G-3' & cytosolic aldehyde dehydrogenase \\
\hline & & 5'-CGA CAG CAA CAC TCT TAC CGA CTT-3' & \\
\hline \multirow[t]{2}{*}{$A D H 1$} & 854068 & 5'-TGC TGC TGG TGG TCT AGG TTC TT-3' & alcohol dehydrogenase \\
\hline & & 5'-GAG ATG GAC TTG ACG ACT TGG TTG A-3' & \\
\hline \multirow[t]{2}{*}{$A D H 2$} & 855349 & 5'-ATT AGT TGG TGG TCA CGA AGG TGC C-3' & glucose-repressible alcohol dehydrogenase II \\
\hline & & 5'-CGG TGA TAC CAG CAC ACA AGA TTG G-3' & \\
\hline \multirow[t]{2}{*}{$A D H 3$} & 855107 & 5'-CAT TGT TCA CCA GGC GTG TCC AA-3' & mitochondrial alcohol dehydrogenase isoenzyme III \\
\hline & & 5'-AAA TCA CCG ACT TTC CAG CCC TTG-3' & \\
\hline \multirow[t]{2}{*}{ ADH4 } & 852636 & 5'-TGG TTC TGC TCA CGA CAA TGC TAA G-3' & alcohol dehydrogenase isoenzyme type IV \\
\hline & & 5'-GGG TTA GAG GCG GTG GAA ACA TAA G-3' & \\
\hline \multirow[t]{2}{*}{ ADH5 } & 852442 & 5'-ACG AAG GTG CTG GTG TTG TTG TT-3' & alcohol dehydrogenase isoenzyme $\mathrm{V}$ \\
\hline & & 5'-GGC GTA TTG GAT TGC CAG AGA ACC-3' & \\
\hline
\end{tabular}

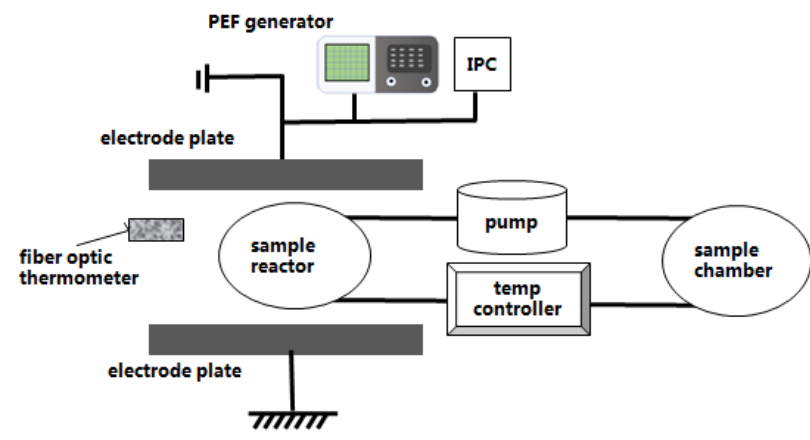

Fig. 1: Schematic diagram of the experimental setup of constant PEF treatment
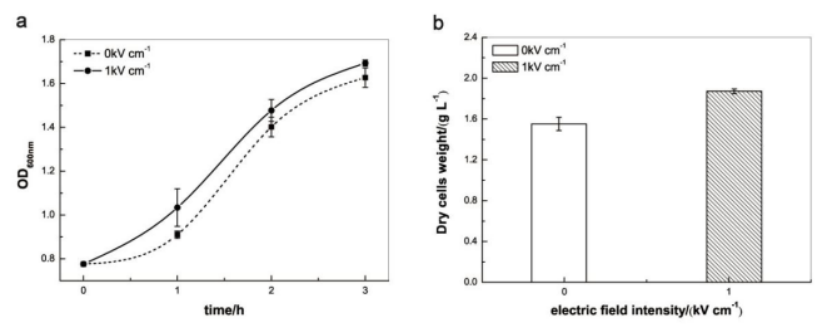

Fig. 2: The effects on growth of $S$. cerevisiae. (a) Growth dynamics of yeast cells. (b) Dry biomass weight. Values represent the average means \pm standard deviations $(n \geq 3)$.

Metabolite analysis of ethanol and glycerol was measured among the fermentation progress, showed in Fig. $3 \mathrm{~b}$ and c. PEF-treated samples increased ethanol production significantly, which increased respectively $12.91 \%$ (1 $\left.\mathrm{kV} \cdot \mathrm{cm}^{-1}\right)$ and $18.26 \%\left(3 \mathrm{kV} \cdot \mathrm{cm}^{-1}\right)$ in ethanol production after $12 \mathrm{~h}$ fermentation (Fig. 3b). A significant increase in ethanol accumulation was observed after $3 \mathrm{~h}$ of fermentation.
The ethanol yield exceeded by 1.18 times $\left(1 \mathrm{kV} \cdot \mathrm{cm}^{-1}\right)$ and 1.43 times $\left(3 \mathrm{kV} \cdot \mathrm{cm}^{-1}\right)$ respectively in treated samples than the control ones after $9 \mathrm{~h}$ fermentation. The trends of glycerol production were similar to ethanol (Fig. 3c). The final glycerol productions were $1.28 \mathrm{~g} \cdot \mathrm{L}^{-1}\left(1 \mathrm{kV} \cdot \mathrm{cm}^{-1}\right)$ and $1.36 \mathrm{~g} \cdot \mathrm{L}^{-1}\left(3 \mathrm{kV} \cdot \mathrm{cm}^{-1}\right)$, which were 2.1 and $7.95 \%$ higher than control samples.

\section{Relative expression levels of glycolysis genes}

In order to reveal the molecule physiological mechanism caused by PEF treatment, relative expression levels of 10 genes (ADH1, ADH2, ADH3, ADH4, ADH5, GPD2, PDC1, PDC6, ALD4, ALD6) were analyzed by RT-qPCR method which involved in glycolysis pathway (Fig. 4).

The expression level of ADH1 was up-regulated by around 4.2 -folds $\left(1 \mathrm{kV} \cdot \mathrm{cm}^{-1}\right)$ and 8.9 -folds $\left(3 \mathrm{kV} \cdot \mathrm{cm}^{-1}\right)$, while ADH2 showed 3.4-folds $\left(1 \mathrm{kV} \cdot \mathrm{cm}^{-1}\right)$ and 5.6-folds (3 $\left.\mathrm{kV} \cdot \mathrm{cm}^{-1}\right)$ than the control samples. The up-regulation of GPD2 has also observed 2.9-folds $\left(1 \mathrm{kV} \cdot \mathrm{cm}^{-1}\right)$ and 3.9-folds $\left(3 \mathrm{kV} \cdot \mathrm{cm}^{-1}\right)$ than the control ones (Fig. 4).

The expression changes in metabolism and fermentation-related genes indicated that PEF stimulated cells by regulating the expression levels of those genes. Cells could respond quickly to PEF stress by enhancing their metabolic bio-synthesis rate. The up-regulation of these genes indicates that the glycolysis reaction and metabolism rate are also enhanced. These results confirm the positive effect of PEF in fermentation capacity.

\section{Discussion}

In this study, variations of physiological behaviors were characterized of $S$. cerevisiae treated by moderate PEF treatment $\left(1 \mathrm{kV} \cdot \mathrm{cm}^{-1}, 3 \mathrm{kV} \cdot \mathrm{cm}^{-1}\right)$. The results showed that 

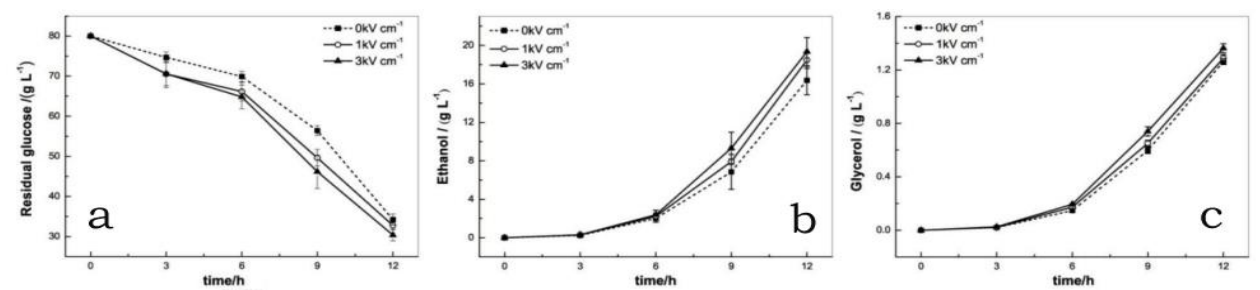

Fig. 3: Effects of PEF on fermentation of S. cerevisiae. (a) Residual glucose in culture, (b) Ethanol concentrations in culture, (c) Glycerol concentrations in culture. Values represent the average means \pm standard deviations $(n \geq 3)$

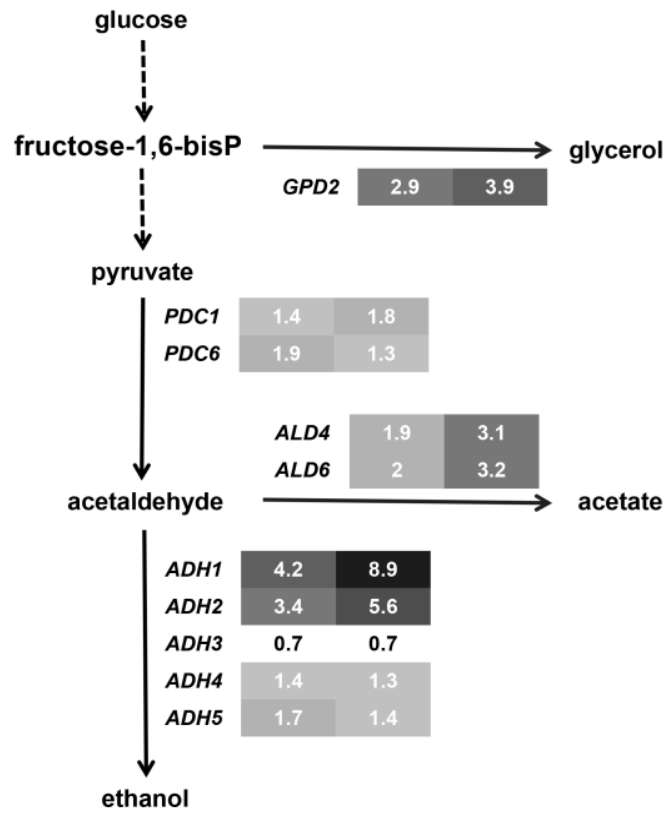

Fig. 4: Schematic representation of the gene expression of the sugar metabolism pathway affected by PEF. The relative fold change shown next to the gene, left $\left(1 \mathrm{kV} \cdot \mathrm{cm}^{-1)}\right.$, and right $(3$ $\mathrm{kV} \cdot \mathrm{cm}^{-1}$ )

fermentation capacity was promoted which manifested in increased consumption of glucose, increased production of metabolites (ethanol and glycerol) and accelerated growth of yeast biomass. The changes in sugar metabolism and fermentation-related gene expression levels also indicated that PEF accelerates yeast fermentation capacity by inducing higher efficiency sugar metabolism. In addition, we also attempted to clarify the correlation between the fermentation phenotype and gene regulation in response to PEF stress.

The electro-poration or electro-permeabilization, which caused by PEF, is a well-recognized physical process in biological cells that influences permeability and diffusion across membranes of cells (Garner 2019). It was reported that the higher uptake of molecules and the rise of ions accumulation in cells under PEF stress (Pankiewicz et al. 2017). In our work, the increasing fermentation capacity after PEF treatment may be due to the increased permeability of the cell membrane. PEF could also directly induce an increase in enzyme activity (Mannozzi et al. 2019; Benede and Molina 2020). We speculate that the dynamic enzymes induced by PEF may also be one of the reasons for the promoted fermentation capacity.

The changes in gene expression are consistent with PEF fermentation phenomena. The glycolysis pathway is the key one in the growth and metabolism of yeast. GPD2 plays a crucial role in osmoregulation and redox balancing, by encoding the enzyme which controls the glycerol formation in the absence of oxygen (Jagtap et al. 2019). Up-regulation of gene GPD2 and higher glycerol yield were observed after PEF stimulation or inducing in this work. Approved that oxidation stress response of genes (SOD1, SOD2 and GSH1) were induced by PEF stimulation, and stress responding caused by PEF was different from heat stress (Tanino et al. 2012). Reported nsPEFs (Nanosecond pulsed electric fields) induced a transient activation of signaling pathways involving mitogen-activated protein kinases (MAPKs) which regulating diverse cellular programs including embryogenesis, proliferation, differentiation and apoptosis (Morotomi-Yano and Akiyama 2011). We speculated that PEF treatment induces higher glucose uptake, and could also directly affect the proliferation of $S$. cerevisiae by regulation of associated-genes.

\section{Conclusion}

In this study we report the positive effects of moderate PEF on both metabolic activity and cell growth of $S$. cerevisiae. Meanwhile, analysis of the transcriptional level of genes involved in glucose metabolism also indicates that PEF contribute to $S$. cerevisiae fermentation. It provides a promising method for fermentation in industrial production. In order to obtain higher production efficiency, it is necessary to determine the optimum conditions in actual production applications. It will be helpful to clarify the complexity responses and mechanism to PEF stress on $S$. cerevisiae. We believe that the combined use of PEF and other techniques such as genetic modification is also feasible to meet the practical production requirements.

\section{Acknowledgement}

This study was funded by the National Natural Science Foundation of China (31401667). 


\section{Author Contributions}

Methods were devised by NJY, WJL and CKF. Experiments were performed by WJL, QSM, SSL and the data analysis by SSL and LZ. The manuscript was written by all authors.

\section{References}

Benede S, E Molina (2020). Chicken egg proteins and derived peptides with antioxidant properties. Foods 9; Article 735

Cai Z, H Riedel, NMT Saw, O Kutuk, I Mewis, H Jager, DI Knorr (2011). Effects of pulsed electric field on secondary metabolism of Vitis vinifera L. cv. Gamay Fréaux suspension culture and exudates. Appl Biochem Biotechnol 164:443-453

Garner AL (2019). Pulsed electric field inactivation of microorganisms: from fundamental biophysics to synergistic treatments. Appl Microbiol Biotechnol 103:7917-7929

Hristov K, U Mangalanathan, M Casciola, ON Pakhomova, AG Pakhomov (2018). Expression of voltage-gated calcium channels augments cell susceptibility to membrane disruption by nanosecond pulsed electric field. Biochim Biophys Acta Biomembr 1860:2175-2183

Jagtap RS, DM Mahajan, SR Mistry, M Bilaiya, R Singh, R KJain (2019). Improving ethanol yields in sugarcane molasses fermentation by engineering the high osmolarity glycerol pathway while maintaining osmotolerance in Saccharomyces cerevisiae. Appl Microbiol Biotechnol 103:1031-1042

Lalwani MA, E Zhao, JL MAvalos (2018). Current and future modalities of dynamic control in metabolic engineering. Curr Opin Biotechnol 52:56-65

Mannozzi C, K Rompoonpol, T Fauster, U Tylewicz, S Romani, RM Dalla, H Jaeger (2019). Influence of pulsed electric field and ohmic heating pretreatments on enzyme and antioxidant activity of fruit and vegetable juices. Foods 8; Article 247

Martens SL, S Klein, RA Barnes, P TrejoSanchez, C Roth, BL Clbey (2020). 600-ns pulsed electric fields affect inactivation and antibiotic susceptibilities of Escherichia coli and Lactobacillus acidophilus. AMB Express 10; Article 55
Mattar JR, MF Turk, M Nonus, NI Lebovka, El Zakhem, E HVorobiev (2015). S. cerevisiae fermentation activity after moderate pulsed electric field pre-treatments. Bioelectrochemistry 103:92-97

Morotomi-Yano K, HK Akiyama (2011). Nanosecond pulsed electric fields activate MAPK pathways in human cells. Arch Biochem Biophys 515:99-106

Najim N, KJ Aryana (2013). A mild pulsed electric field condition that improves acid tolerance, growth, and protease activity of Lactobacillus acidophilus LA-K and Lactobacillus delbrueckii subspecies bulgaricus LB-12. J Dairy Sci 96:3424-3434

Ojha KS, TJ Mason, CP O'Donnell, J Kerry, BK PTiwari (2017). Ultrasound technology for food fermentation applications. Ultrason Sonochem 34:410-417

Pankiewicz U, M Sujka, R Kowalski, A Mazurek, MJ Wlodarczyk-Stasiak (2017). Effect of pulsed electric fields (PEF) on accumulation of selenium and zinc ions in Saccharomyces cerevisiae cells. Food Chem 221:1361-1370

Santos LO, RM Alegre, C Garcia-Diego, J Cuellar (2010). Effects of magnetic fields on biomass and glutathione production by the yeast Saccharomyces cerevisiae. Process Biochem 45:1362-1367

Semkiv MV, KV Dmytruk, C Abbas, AA ASibirny (2017). Metabolic engineering for high glycerol production by the anaerobic cultures of Saccharomyces cerevisiae. Appl Microbiol Biotechnol 101:4403-4416

Seong YJ, H Park, J Yang, SJ Kim, W Choi, K Kim, YC HPark (2017). Expression of a mutated SPT15 gene in Saccharomyces cerevisiae enhances both cell growth and ethanol production in microaerobic batch, fed-batch, and simultaneous saccharification and fermentations. Appl Microbiol Biotechnol 101:3567-3575

Tanino T, S Sato, M Oshige, T Ohshima (2012). Analysis of the stress response of yeast Saccharomyces cerevisiae toward pulsed electric field. J Electrostat 70:212-216

Yin X, S Qiao, J Zhou (2015). Using electric field to enhance the activity of anammox bacteria. Appl Microbiol Biotechnol 99:6921-6930

Zhao YM, M Alba, D Sun, B W Tiwari (2019). Principles and recent applications of novel non-thermal processing technologies for the fish industry-a review. Crit Rev Food Sci Nutr 59:728-742 